\title{
Formation of granular intensity fluctuations on the Sun
}

\author{
E. Pérez Rodríguez and F. Kneer \\ Universitäts-Sternwarte, Geismarlandstraße 11, 37083 Göttingen, Germany \\ Received 24 June 2002 / Accepted 27 August 2002

\begin{abstract}
We deal with granular intensity fluctuations in the Sun and how they are caused by temperature fluctuations. We calculate temperature response functions $R F_{\mathrm{T}}(\lambda, z)$ in the solar photosphere. We apply the Diagonal Element Lambda Operator (DELO) method to calculate the response functions for the continuum $(\lambda \lambda=380 \mathrm{~nm}-2.5 \mu)$. LTE is assumed throughout. It is demonstrated that, within the framework of the linear approximation, the $\mathrm{H}^{-}$continuum absorption plays an important rôle for the formation of the fluctuations in continuum images. It counteracts substantially the intensity fluctuations caused by variations of the Planck function. The temperature response functions are used to calculate, for three models of temperature fluctuations, the continuum intensity contrasts and their formation height for the above wavelength range.
\end{abstract}

Key words. Sun: photosphere - Sun: granulation - radiative transfer

\section{Introduction}

It is a widespread believe that the fluctuation of the granular intensity pattern on the Sun can be approximated by the simple relation $\delta I_{\lambda} / I_{\lambda}=\delta B_{\lambda} / B_{\lambda}$ with $B_{\lambda}$ being the Planck function. Thus, in the linear approximation with $\delta B_{\lambda} \propto \delta T$, the observation of $\delta I_{\lambda}$ would give a good proxy to $\delta T$ at some optical depth $\tau_{\lambda} \approx \cos \theta$, the latter according to the Eddington-Barbier relation.

Such reasonning falls short on two grounds: 1) It neglects the opacity changes due to temperature changes. 2) Simulations of the granular convection (e.g. Solanki et al. 1996; Stein \& Nordlund 1998, 2000; Steffen \& Holweger 2002, and references therein) demonstrate that the excursions $\delta T$ can be very large and thus $\delta B_{\lambda} \propto \delta T$ is no longer valid and that the continuous optical depth $\tau_{\text {cont }}=1$ is very rugged. Beyond the granular intensity contrast, Steffen \& Holweger (2002) have demonstrated with numerical simulations that the equivalent widths of spectral lines depend non-linearly on the temperature fluctuations.

We shall not discuss non-linearities here. We aim at discussing the influence of the continuum opacity and its temperature sensitivity on the fluctuation of the emergent intensities. In the solar atmosphere, as in atmospheres of late-type stars, the continuum opacity in the visible and infra-red spectral range is mainly due to the $\mathrm{H}^{-}$ion. We restrict ourselves to the linear regime from which one can already gain insight into the formation of intensity fluctuations, without the need to refer to complex non-linear calculations. For this purpose, it is appropriate to calculate temperature response functions $\operatorname{RF}_{\mathrm{T}}(\lambda, z)$ for a given mean solar atmospheric model.

Response functions are a means to obtain a first order (linear) approximation on the height range in the solar atmosphere

Send offprint requests to: F. Kneer,

e-mail: kneer@uni-sw.gwdg.de in which fluctuations of certain atmospheric parameters (temperature, velocity, ....) influence observed fluctuations. They were introduced by Mein (1971) and are widely in use nowadays (see e.g. Eibe et al. 2001 and references therein and references in Kneer \& Nolte 1991). In addition, response functions are applied in inversion codes to obtain heights of formation in which the atmospheric model parameters are to be tuned iteratively for a best fit of modelled, synthetic intensities to the observations (e.g. Ruiz Cobo \& del Toro Iniesta 1994; Bellot Rubio et al. 2000; Borrero \& Bellot Rubio 2002 and references there).

We will outline the method of the calculations of temperature response functions in the next section. This will include both continuum and line opacities. Some results concerning the formation of continuum fluctuations upon temperature variations will be presented in Sect. 3. Section 4 conludes the paper.

\section{Temperature response functions}

Given an average atmosphere, one asks, very generally, how big the change $\delta I_{\lambda}$ of the average intensity $I_{\lambda}$ would be if a certain atmospheric parameter $P(z)$, e.g. the temperature $T(z)$, is changed by a small amount $\delta P(z)$ in the height range $(z, z+$ $\delta z)$. In the linear regime, one has $\delta I_{\lambda} \propto \delta P(z)$ and the response function can be defined as

$$
R F_{P}(\lambda, z)=\frac{\delta I_{\lambda}}{\delta P(z) \delta z}
$$

The total intensity change $\Delta I_{\lambda}$ by a height dependent $\delta P(z)$ will then be

$\Delta I_{\lambda}=\sum R F_{P}(\lambda, z) \delta P(z) \delta z$

where the summation is taken over all heights. 
We shall deal with the solar photosphere up to a height of approximately $500 \mathrm{~km}$ (above $\tau_{5000}=1$ ). We will assume LTE throughout. Thus, the atomic level populations and the ionization equilibria follow from the Boltzmann-Saha statistics and the source function is given by the Planck function, $S_{\lambda}(z)=B_{\lambda}(T(z))$. As a consequence, the changes of the source function and of the opacities upon a (small) temperature perturbation can be evaluated analytically, to a wide extent. A detailed description of the assumptions and the calculations is found in Pérez Rodríguez (2001).

\subsection{Model atmosphere}

The average solar atmosphere is represented by the model $\mathrm{C}$ of Vernazza et al. (1981), commonly named VALC model. The temperature $T(m)$, with $m=$ column mass density, and the microturbulent velocity $\xi_{\mathrm{t}}(m)$ serve as input for a selfconsistent calculation of the complete model in hydrostatic equilibrium, i.e. fulfilling

$p_{\text {tot }}=g m+p_{0}$.

Here, the total pressure is $p_{\text {tot }}=p_{\text {gas }}+\frac{1}{2} \rho \xi_{t}^{2}, p_{\text {gas }}=$ gas pressure, $\rho=$ mass density, $g=$ surface gravity, and $p_{0}=$ pressure at the top of the atmosphere $(\approx 0)$.

For solar abundances, the gas pressure and the mass density are calculated from an initial guess of the electron pressure with the procedure given by Mihalas (1970). The electron pressure is iterated with the secant method until the hydrostatic equilibrium, Eq. (3), is fulfilled to a relative accuracy of $10^{-5}$ yielding the gas pressure and mass density to the same accuracy.

The geometric height, $z$, is then obtained from

$\mathrm{d} m=-\rho \mathrm{d} z$.

\subsection{Opacities}

In the solar photosphere, in the wavelength range of interest here, $\lambda \lambda=380 \mathrm{~nm}-2.5 \mu$, the continuum absorption is overwhelmingly due to $\mathrm{H}^{-}$bound-free and free-free transitions. The small contributions from $\mathrm{H}$ free-free transitions and from the Paschen-, Bracket- and Pfund bound-free transitions of hydrogen are also included. We use the routines from the LINEARA code by Auer et al. (1972). There polynomial fits in wavelength $\lambda$ and temperature $T$, sometimes also in $\Theta=5040 / T$, to the opacities are taken from Carbon \& Gingerich (1969). The Gaunt factors are calculated, also via analytical expressions in $\lambda$ and $\Theta$, following Mihalas (1967). With known continuum opacities it is now easy to locate the position in the atmosphere where $\tau_{5000}=1$. Following common use we define $z=0$ at this position by shifting the geometric height from Eq. (4) by an appropriate amount.

The present contribution is embedded in a broader work which includes the treatment of spectral lines. We therefore comment on line opacities. They are calculated using the (solar) abundance of the element of the line under consideration, the lower level excitation energy, the partition functions of the relevant ionization states, the oscillator strength, and the collisional damping. For the latter we use van der Waals damping according to Unsöld (1955). As Borrero \& Bellot Rubio (2002) we include enhancement factors to the damping constants to fit the calculated wings to those of the Fourier Transform Spectrometer Atlas by Brault \& Neckel (cited by Neckel 1999) The absorption profile is given by the Voigt function calculated from the complex error function after Hui et al. (1978). The partition functions are considered, as an approximation for the response functions of lines, independent of temperature.

\subsection{Radiative transfer}

We solve the radiative transfer equation for the specific intensity $I_{\lambda}$ in a plane parallel atmosphere

$\mu \frac{\mathrm{d} I_{\lambda}}{\mathrm{d} z}=-\kappa_{\lambda}\left(I_{\lambda}-S_{\lambda}\right)$

where $\mu=\cos \theta$ and $\kappa_{\lambda}$ is the absorption coefficient. We adapt the DELO (diagonal element lambda operator) method by Rees et al. (1989) to the much simpler case when the radiative transfer problem for polarized light, as in their work, is reduced to solving only for the specific intensity in the absence of polarization. Henceforth, we consider only radiation propagating perpendicularly to the "surface", i.e. we take $\mu=1$.

The emergent intensity is

$$
\begin{aligned}
I_{\lambda}\left(\tau_{\lambda}=0\right)= & \int_{z_{\mathrm{ND}}}^{z_{1}} O_{\lambda}\left(z_{1}, z^{\prime}\right) \kappa_{\lambda}\left(z^{\prime}\right) S_{\lambda}\left(z^{\prime}\right) \mathrm{d} z^{\prime} \\
& +O_{\lambda}\left(z_{1}, z_{\mathrm{ND}}\right) I_{\lambda}^{+}
\end{aligned}
$$

The integration limits are $z_{\mathrm{ND}}$ (= bottom, deep in the atmosphere) and $z_{1}\left(=\right.$ top, at $\left.\tau_{\lambda}=0\right) . I_{\lambda}^{+}$is the intensity irradiated at the bottom. $O_{\lambda}\left(z_{1}, z^{\prime}\right)=\exp \left[-\tau_{\lambda}\left(z^{\prime}\right)\right]$ is the attenuation factor, in the more general case of transfer of polarized light the attenuation operator (Landi Degl'Innocenti \& Landi Degl'Innocenti 1985).

We omit, for brevity, the index for the wavelength dependence. $\tau_{k}$ are the optical depths on the corresponding geometric grid $\left[z_{1}, z_{2}, \ldots, z_{k}, \ldots, z_{\mathrm{ND}}\right]$. We use the notations

$\mathcal{D}_{k}=\mathrm{e}^{-\tilde{\delta}_{k}}$ with $\tilde{\delta}_{k}=\tau_{k+1}-\tau_{k}$,

and approximate the source function in the interval $\left[\tau_{k}, \tau_{k+1}\right]$ by a linear dependence

$S(\tau)=\left[\left(\tau_{k+1}-\tau\right) S_{k}+\left(\tau-\tau_{k}\right) S_{k+1}\right] / \tilde{\delta}_{k}$.

The emergent intensity $I(\tau=0)$ is then obtained from the recursive formula (Rees et al. 1989)

$I\left(\tau_{k}\right)=\mathcal{P}_{k}+\mathcal{D}_{k} I\left(\tau_{k+1}\right)$

where

$\mathcal{P}_{k}=\left(F_{k}-G_{k}\right) S_{k}+G_{k} S_{k+1}$,

$F_{k}=1-\mathcal{D}_{k}$,

and

$G_{k}=\left[1-\left(1+\tilde{\delta}_{k}\right) \mathcal{D}_{k}\right] / \tilde{\delta}_{k}$ 


\subsection{Response functions}

We introduce a small perturbation $\delta P$ of an atmospheric parameter $P$ and linearize the transfer equation Eq. (5) (for $\mu=1$ ) which gives

$\frac{\mathrm{d}}{\mathrm{d} z} \delta I=-\kappa(\delta I-\delta S)-\delta \kappa(I-S)$,

or

$\frac{\mathrm{d}}{\mathrm{d} z} \delta I=-\kappa\left(\delta I-\delta S^{*}\right)$

where

$\delta S^{*}=\delta S-\frac{\delta \kappa}{\kappa}(I-S)$.

The attenuation factor $O\left(z_{1}, z_{i}\right)$ can be factorized. With

$O\left(z_{k}, z_{k+1}\right)=\mathcal{D}_{k}=\mathrm{e}^{-\tilde{\delta}_{k}}$

(Eq. (7)) one obtains

$O\left(z_{1}, z_{i}\right)=\mathcal{D}_{1} \mathcal{D}_{2} \ldots \mathcal{D}_{i-1}$.

If we perturb the atmosphere only in a small height range $\delta z$ about $z_{i}$, that is, if we have no contributions from other heights to the transfer equation Eq. (14), the solution is

$\delta I=O\left(z_{1}, z_{i}\right) \kappa \delta S^{*} \delta z$,

and the response function follows as

$R F_{P}\left(\lambda, z_{i}\right)=\mathcal{D}_{1} \mathcal{D}_{2} \ldots \mathcal{D}_{i-1} \kappa_{\lambda}\left(z_{i}\right) \delta S_{\lambda}^{*}\left(z_{i}\right) / \delta P\left(z_{i}\right)$.

We take the temperature $T$ as the perturbed parameter and have (in LTE)

$\kappa_{\lambda} \delta S_{\lambda}^{*}=\kappa_{\lambda} \frac{\partial B_{\lambda}(T)}{\partial T} \delta T-\frac{\partial \kappa_{\lambda}}{\partial T}\left(I_{\lambda}-B_{\lambda}(T)\right) \delta T$

and thus

$R F_{\mathrm{T}}\left(\lambda, z_{i}\right)=O_{\lambda}\left(z_{1}, z_{i}\right)\left[\kappa_{\lambda} \frac{\partial B_{\lambda}(T)}{\partial T}-\frac{\partial \kappa_{\lambda}}{\partial T}\left(I_{\lambda}-B_{\lambda}(T)\right)\right]$.

We note:

1. The attenuation factors $O_{\lambda}\left(z_{1}, z_{i}\right)$ as well as the intensities $I_{\lambda}\left(z_{i}\right)$, thus also the differences $I_{\lambda}-B_{\lambda}$, are already known from the calculation of the unperturbed intensities;

2. the derivative of the absorption coefficient appears in Eq. (21) which, as we will see, makes an important contribution to the response function. Increased opacity due to increased temperature reduces the response function, thus reduces (partly) the contrast of the observable intensity fluctuations.

We calculate the derivatives of the opacities preserving the horizontal pressure balance at each geometric height, as in the reasonning for the Schwarzschild criterion for convective instability, i.e. we require

$\delta p_{\text {tot }}=\delta p_{\text {gas }}+\delta \rho \xi_{\mathrm{t}}^{2}=0$

with

$\delta p_{\text {gas }}=\delta n_{\text {tot }} k T+n_{\text {tot }} k \delta T$, where $n_{\text {tot }}$ is the total particle density and $k$ is the Boltzmann constant. This pressure constancy neglects the pressure fluctuations in the convection process. Horizontal pressure imbalance is necessary to drive horizontal flows and to deflect the horizontal flows downward in the intergranular regions (e.g. Stein \& Nordlund 1998). However, it has been shown by Kneer \& Nolte (1991) that the influence of pressure fluctuations on the emergent intensities, at least continuum intensities, is a factor of 5-10 smaller than that of temperature fluctuations.

The variation of the mass density is expressed by

$\delta \rho=1.4 m_{\mathrm{H}} \delta n_{\mathrm{H}}$

with $m_{\mathrm{H}}=$ mass of the hydrogen atom and $n_{\mathrm{H}}=$ number density of all hydrogen nuclei. From the other elements only helium, with an abundance of 0.1 in particles, contributes substantially to the mass density, hence the factor 1.4.

In the VALC model, up to the temperature minimum, the electron density $n_{\mathrm{e}}$ is approximated, within $20 \%$ accuracy in high layers and within $1 \%$ accuracy in deep layers, by the formula

$n_{\mathrm{e}}=n_{\mathrm{p}}+810^{-5} n_{\mathrm{H}}$,

where $n_{\mathrm{p}}$ is the proton density.

Although we shall not expand on temperature response functions for spectral lines below, we give here for completeness the temperature derivatives of the Voigt function $H(a, v)$, where $a=\Gamma /\left(4 \pi \Delta v_{\mathrm{D}}\right)$ and $v=\left(v-v_{0}\right) / \Delta v_{\mathrm{D}} . \Gamma$ is the damping constant and $\Delta v_{\mathrm{D}}$ is the Doppler width, expressed in frequencies. We have

$\frac{\partial H}{\partial T}=\frac{\partial H}{\partial a} \frac{\partial a}{\partial T}+\frac{\partial H}{\partial v} \frac{\partial v}{\partial T}$

and with the Faraday function $F(a, v)$, from e.g. Ruiz Cobo \& del Toro Iniesta (1994, see also Hui et al. 1978),

$\frac{\partial H}{\partial a}=2 a H(a, v)+4 v F(a, v)-\frac{2}{\sqrt{\pi}}$

$\frac{\partial H}{\partial v}=4 a F(a, v)-2 v H(a, v)$.

The derivative of the absorption coefficients $\kappa_{\lambda}$ with respect to temperature $T$ can now be calculated completely analytically.

\section{Granular intensity fluctuations}

In this Section, we present results for continuum intensity fluctuations in a wide spectral range, $380 \mathrm{~nm}$ to $2.5 \mu$. We have extended the calculations into the IR region because there too, as in the visible, spectral features with excellent diagnostic properties can be observed. We mention the He I line at $1.083 \mu$, the two Zeeman sensitive Fe $\mathrm{I}$ lines at $1.565 \mu$, the $\mathrm{H}^{-}$opacity minimum at $1.655 \mu$, and the $\mathrm{CO}$ rotation-vibration band with $\Delta v=2(v=$ vibration quantum number $)$ at $2.3 \mu$. The capability of IR observations will be much improved with new, large solar telescopes and with new detectors. 


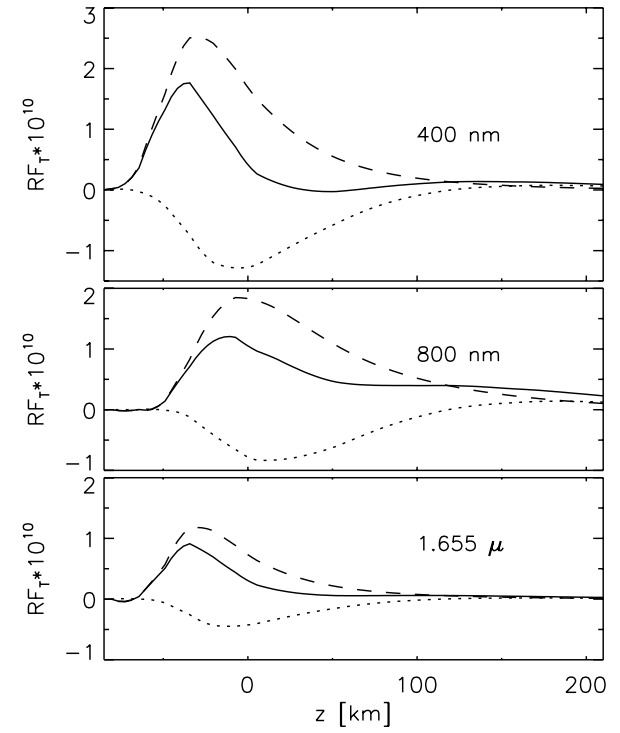

Fig. 1. Temperature response functions $R F_{\mathrm{T}}(\lambda, z)$ for the continuum at three wavelengths. Dashed curves: $R F_{\mathrm{T}}$ from the variation of the Planck function (=source function) alone; dotted: $R F_{\mathrm{T}}$ from the variation of the (continuum) opacity alone; solid: sum of dashed and dotted, i.e. full $R F_{\mathrm{T}}$. The units of the ordinates are $\operatorname{erg~} \mathrm{cm}^{-2} \mathrm{~Hz}^{-1}$ sterad $^{-1} \mathrm{~K}^{-1} \mathrm{~km}^{-1}$.

\subsection{Temperature response functions}

Figure 1 depicts temperature response functions $R F_{\mathrm{T}}(\lambda, z)$ (for the continuum) at three wavelengths, in the blue range at $400 \mathrm{~nm}$, at $800 \mathrm{~nm}$ close to the maximum of the $\mathrm{H}^{-}$opacity (cf. Allen 1963), and at the minimum of the $\mathrm{H}^{-}$opacity at $1.655 \mu$. The response functions are presented in a way to show separately the effects of source function fluctuations alone (first term in the brackets at r.h.s. of Eq. (21), dashed in Fig. 1) and of opacity fluctuations alone (second term in Eq. (21), dotted). We discuss several properties:

1. The amplitudes of the response functions decrease with increasing wavelength. This is not generally the case. Inspection of the other calculated response functions shows the maximum amplitude for the source function fluctuations alone at a wavelength around $500 \mathrm{~nm}$. Yet the total response function, i.e. including opacity fluctuations, possesses indeed its maximum amplitude near $400 \mathrm{~nm}$. This behaviour can be understood from Eq. (21). It results from the interplay between the wavelength dependences of the attenuation factors $O_{\lambda}\left(z_{1}, z_{i}\right)$, of the opacity $\kappa_{\lambda}$ and its derivatives, of $\partial B_{\lambda}(T) / \partial T$, and of the factor $\left(I_{\lambda}-B_{\lambda}(T)\right)$ in the second term r.h.s. of Eq. (21). $\kappa_{\lambda}$ increases with increasing wavelength til $800 \mathrm{~nm}$, and so does $\partial B_{\lambda}(T) / \partial T$ whose wavelength position of its maximum depends on temperature. Yet the attenuation factor moves with increasing wavelength, up to $800 \mathrm{~nm}$, the maximum of the response function to larger atmospheric heights with lower temperatures. At wavelengths larger than about $1 \mu$, both terms in the brackets of Eq. (21) decrease, overriding the increase of the attenuation factor.

2. The contributions from the opacity have a strong influence as already noted by Kneer et al. (1980). They are negative at

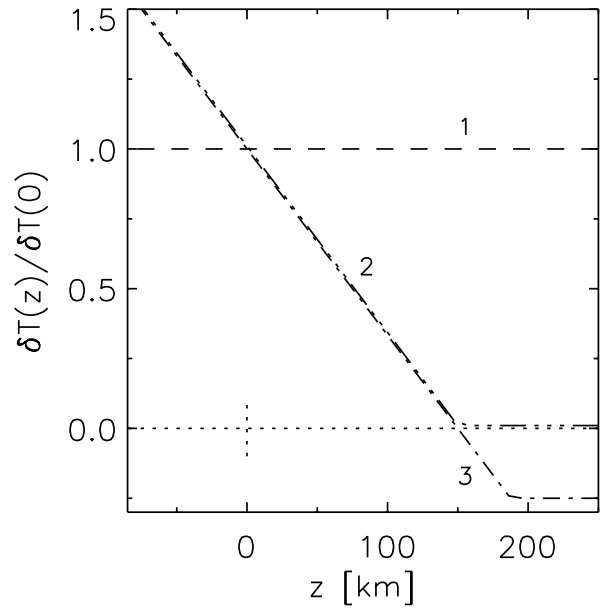

Fig. 2. Three models of the (rms) temperature fluctuation.

low atmospheric heights. There, a strong increas of the $\mathrm{H}^{-}$ opacity occurs due to increased electron density from hydrogen ionization. At larger heights, where hydrogen does not serve as an important electron donor, the $\mathrm{H}^{-}$opacity decreases due to ionization of $\mathrm{H}^{-}$itself and to the condition of constant pressure, i.e. decreasing particle density. There, the opacity contribution is positive and gives rise to extended "wings" of the temperature response functions for the continuum which reach to heights above $200 \mathrm{~km}$.

\subsection{Intensity contrast}

To obtain intensity fluctuations we apply height dependent temperature fluctuations $\delta T(z)$ according to Eq. (2). Since the emergent intensity fluctuations depend linearly on $\delta T(z)$ its actual amplitude is of no importance. Figure 2 gives three "models": (1) with $\delta T$ independent of atmospheric height, (2) with $\delta T=0$ above $150 \mathrm{~km}$ and increasing below $150 \mathrm{~km}$ linearly towards subphotospheric layers, and (3) similar as (2) but decreasing to a minimum of $\delta T(z) / \delta T(0)=-0.25$ for $z>150 \mathrm{~km}$. All three models possess the same $\delta T$ at $z=0$.

Model 2, which we consider more realistic than model 1, is suggested by the work of Komm et al. (1990). They find that the intensity contrast measured in the wings of strong absorption lines looses its coherence with continuum contrasts in the mid-photosphere. Temperature inversions in higher layers, as in the third model of Fig. 2, are found by, e.g., Holweger \& Kneer (1989), Ruiz Cobo et al. (1996), Gadun et al. (2000), and Borrereo \& Bellot Rubio (2002).

Figure 3 , then, gives the contrasts $\Delta I_{\lambda} / \bar{I}_{\lambda}$ as functions of wavelength for models 1 and 2 . The results for model 3 are few percent lower than those for model 2, but otherwise very similar. Thus, we do not show them here. All the contrasts are normalized such that we have the value of 0.15 at $550 \mathrm{~nm}$ from model 2 (indicated by $\diamond$ in Fig. 3). This contrast comes close to that measured in speckle reconstructions by de Boer (1995).

To demonstrate the influence of the opacity changes upon temperature fluctuations we give in Fig. 3 the contrasts separately for the fluctuations of the source function alone (dashed 


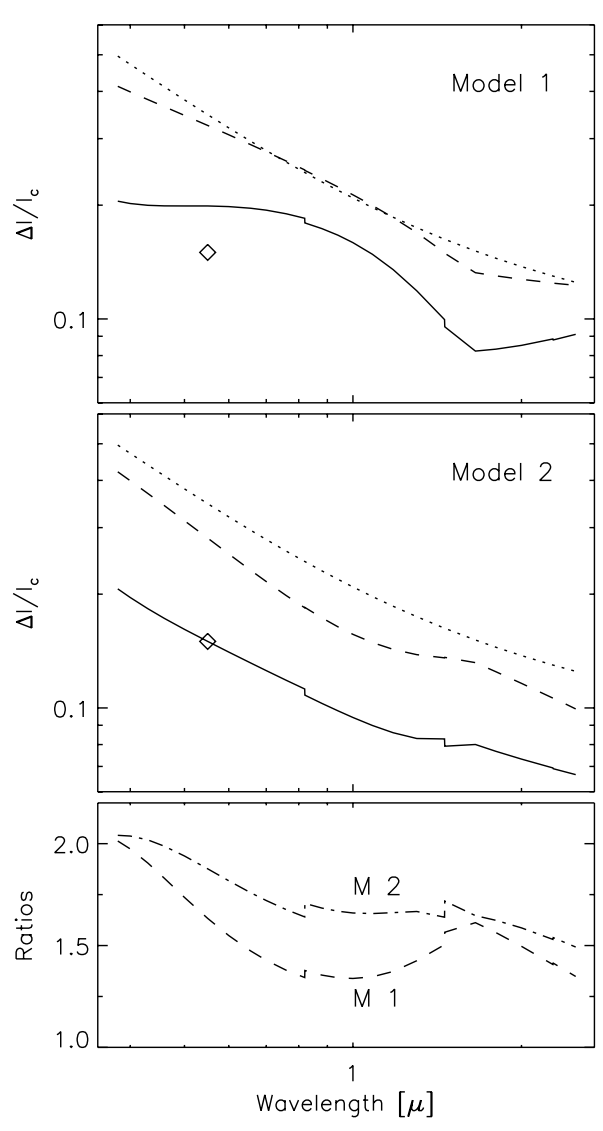

Fig. 3. Wavelength dependence of continuum intensity contrasts $\Delta I_{\mathrm{c}} / \bar{I}_{\mathrm{c}}$ calculated from the temperature fluctuations of model 1 (upper panel) and model 2 (middle panel) of Fig. 2. The contrasts are normalized to give $\Delta I_{\mathrm{c}} / \bar{I}_{\mathrm{c}}=0.15$ at $550 \mathrm{~nm}$ for model $2(\diamond)$. Dashed: from the variation of the Planck function alone; solid: including the opacity dependence; dotted: intensity contrasts from $\delta B / B$ of Eq. (29) with a temperature of $6250 \mathrm{~K}$ and with the $T(z=0)$ as in the models of Fig. 2. Lower panel: ratios of the contrasts from Planck function fluctuations alone to contrasts from the full response functions.

curves) and including the opacity effects (solid curves). The dotted curves represent the wavelength dependence of

$\frac{\delta B_{\lambda}(T)}{B_{\lambda}(T)}=\frac{1}{B_{\lambda}(T)} \frac{\partial B_{\lambda}}{\partial T} \delta T$

with $T=6250 \mathrm{~K}$ and the same $\delta T$ as for the models $1-3$ at $z=0$. The contrasts including the opacity effects from model 2 have very much the same wavelength dependence as that given from the above Eq. (29). Measuring such a wavelength dependence, it would be tempting, but misleading, to ascribe some rms intensity fluctuations to the fluctuation of the source function (= Planck function) and to calculate the temperature fluctuation from Eq. (29). The lower panel of Fig. 3 gives the ratios of the contrasts calculated for the source function fluctuations alone to those including opacity effects. The latter yield a contrast reduction by a factor 1.5-2.0 for model 2 .

Apart from short wavelengths, the contrasts in model 1 are larger than those in model 2 because in the former the atmospheric layers above $z=0 \mathrm{~km}$ contribute stronger than in the latter. Especially above $z=150 \mathrm{~km}$, model 1 together with the extended response functions gives high contrasts. It is also

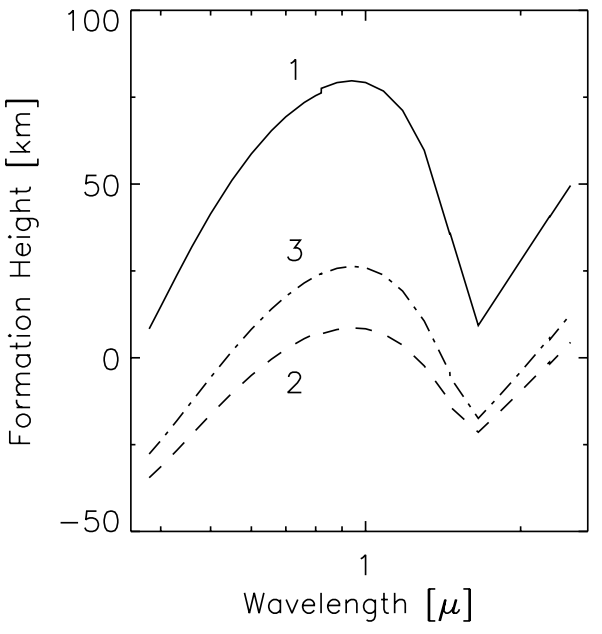

Fig. 4. Wavelength dependence of the average formation heights $\bar{z}$ of continuum contrasts for the three models $\delta T(z)$ from Fig. 2 according to Eq. (30).

due to these high reaching response functions that the contrasts for model 1 increase above $1.655 \mu$ towards still longer wavelengths. We note in passing that one sees in the contrasts, which include the opacity effects, the Paschen- and Bracket boundaries of the hydrogen bound-free transitions at $821 \mathrm{~nm}$ and at $1.459 \mu$, respectively.

\subsection{Formation height}

We may now ask at which average height $\bar{z}_{\lambda}$ in the atmosphere the intensity fluctuations (in the continuum) are formed. We use two definitions, one which includes the structure of the temperature fluctuations and one which does not, i.e.

$\bar{z}_{1, \lambda}=\frac{\int R F_{\mathrm{T}}(z, \lambda)|\delta T(z)| z \mathrm{~d} z}{\int R F_{\mathrm{T}}(z, \lambda)|\delta T(z)| \mathrm{d} z}$,

and

$\bar{z}_{2, \lambda}=\frac{\int R F_{\mathrm{T}}(z, \lambda) z \mathrm{~d} z}{\int R F_{\mathrm{T}}(z, \lambda) \mathrm{d} z}$.

For temperature fluctuations independent of height, as in model 1, Eq. (30) is reduced to Eq. (31), i.e. one obtains the center of gravity of the response functions and $\bar{z}_{1, \lambda}=\bar{z}_{2, \lambda}$. Not taking the absolute values of the temperature fluctuations in Eq. (30) would give a meaningless results in the case of model 3. A negative rms temperature fluctuation at high atmospheric layers would lower the average formation height. Including the temperature fluctuation in the calculation of the average height makes sense because only there where temperature variations do occur the intensity variations can be formed.

Figure 4 depicts the formation heights of continuum intensity fluctuations for the three models. The wavelength dependence shows clearly the opacity dependence. We consider the formation heights of models 2 and 3 (between approximately $-35 \mathrm{~km}$ and $+25 \mathrm{~km}$ ) as more realistic than those of model 1 because the latter are just centers of gravity of very extended contribution functions. Yet, generally, the formation heights 
should not be taken too literally. Even in this limited context of linear approximations, it is better to test models of (granular) temperature fluctuations by calculating the emergent intensities by means of the response functions and compare them with observations.

\section{Conclusion}

We have presented temperature response functions $R F_{\mathrm{T}}(z, \lambda)$ for the continua in the wavelength range of $380 \mathrm{~nm}-2.5 \mu$. They were calculated from the VALC solar photospheric model (Veranzza et al. 1981) and by linearizing the transfer equation using LTE. The differentiation of the opacities with respect to temperature could be performed analytically. The response functions were used to calculate, in linear approximation, granular intensity contrasts and formation heights in the above wavelength range. We emphasized the importance of opacities, especially that of the $\mathrm{H}^{-}$ion, which reduce the intensity contrast by up to a factor of two compared to calculations which neglect the opacity effects.

Response functions neglect non-linear effects which occur when the temperature fluctuations are large. We thus reiterate that such calculations can give only first order impressions of the correct fluctuations of atmospheric parameters which cause the granular intensity pattern. Yet, response functions are often helpful in preparing and analysing observations to have a first order conception where and how spectral features are formed and how they react on fluctuations of the atmospheric structure.

Acknowledgements. Most of this work was performed while EPR was studying at the Göttingen University with support from the ERASMUS program of the European Union.

\section{References}

Allen, C. W. 1963, Astrophysical Quantities, 2nd ed. (London: The Athlone Press), 99

Auer, L. H., Heasley, J. N., \& Milkey, R. W. 1972, A Computational Program for the Solution of Non-LTE Transfer Problems by the Complete Linearization Method, Kitt Peak National Observatory, Contrib. No. 555
Bellot Rubio, L. R., Ruiz Cobo, B., \& Collados, M. 2000, ApJ, 535, 475

Borrero, J. M., \& Bellot Rubio, L. R. 2002, A\&A, 385, 1056

Carbon, D. F., \& Gingerich, O. 1969, The Grid of Model Stellar Atmospheres from $4000 \mathrm{~K}$ to $10000 \mathrm{~K}$. In Proc. of 3rd Harvard-Smithsonian Conf. on Stellar Atmospheres, Theory and Observations of Normal Stellar Atmospheres, ed. O. Gingerich (MIT Press), 377

de Boer, C. R. 1995, A\&AS, 114, 387

Eibe, M. T., Mein, P., Roudier, Th., \& Faurobert, M. 2001, A\&A, 371, 1128

Gadun, A. S., Hanslmeier, A., Kucera, A., Rybák, J., \& Wöhl, H. 2000, A\&A, 363, 289

Holweger, H., \& Kneer, F. 1989, Spatially resoved Spectra of Solar Granules, in Solar and Stellar Granulation, ed. R. J. Rutten, \& G. Severino (Kluwer, Dordrecht), 173

Hui, A. K., Armstrong, B. H., \& Wray, A. A. 1978, J. Quant. Spectrosc. Radiat. Transfer, 19, 509

Kneer, F., \& Nolte, U. 1994, A\&A, 286, 309

Kneer, F., Mattig, W., Nesis, A., \& Werner, W. 1980, Sol. Phys., 68, 31

Komm, R., Mattig, W., \& Nesis, A. 1990, A\&A, 239, 340

Landi Degl'Innocenti, E., \& Landi Degl'Innocenti, M. 1985, Sol. Phys., 97, 239

Mein, P. 1971, Sol. Phys., 20, 3

Mihalas, D. 1967, ApJ, 149, 169

Mihalas, D. 1970, Stellar Atmospheres (Freeman, San Francisco), 72

Neckel, H. 1999, Sol. Phys., 184, 421

Pérez Rodríguez, E. 2001, Diploma thesis, Göttingen university

Rees, D. E., Murphy, G. A., \& Durrant, C. J. 1989, ApJ, 339, 1093

Ruiz Cobo, B., \& del Toro Iniesta, J. C. 1994, ApJ, 283, 19

Ruiz Cobo, B., del Toro Iniesta, J. C., Rodríguez Hidalgo, I., \& Sánchez Almeida, J. 1996, in 9th Cambridge Workshop, Cool Stars, Stellar Systems and the Sun, ASP Conf. Ser., 109, 155

Solanki, S. K., Rüedi, I., Bianda, M., \& Steffen, M. 1996, A\&A, 308, 623

Stein, R. F., \& Nordlund, ̊̊ 1998, ApJ, 499, 914

Stein, R. F., \& Nordlund, Å 2000, Sol. Phys., 192, 91

Steffen, M., \& Holweger, H. 2002, A\&A, 387, 258

Unsöld, A. 1955, Physik der Sternatmosphären, 2nd ed. (Springer, Heidelberg), 333

Vernazza, J. E., Avrett, E. H., \& Loeser, R. 1981, ApJS, 45, 635 